PPPL-3463

PPPL-3463

UC-70

Effect of Plasma Rotation on Sawtooth Stabilization by Beam Ion

by

N.N. Gorelenkov, M.F.F. Nave, R. Budny, C.Z. Cheng,

G.Y. Fu, J. Hastie, J. Manickam, and W. Park

June 2000

$\int D \sqrt{D} \sqrt{5} \int \sqrt{\zeta} \int \begin{aligned} & \text { PAINCETON } \\ & \text { PLABMA PHYSICS } \\ & \text { LABOAATOAY }\end{aligned}$

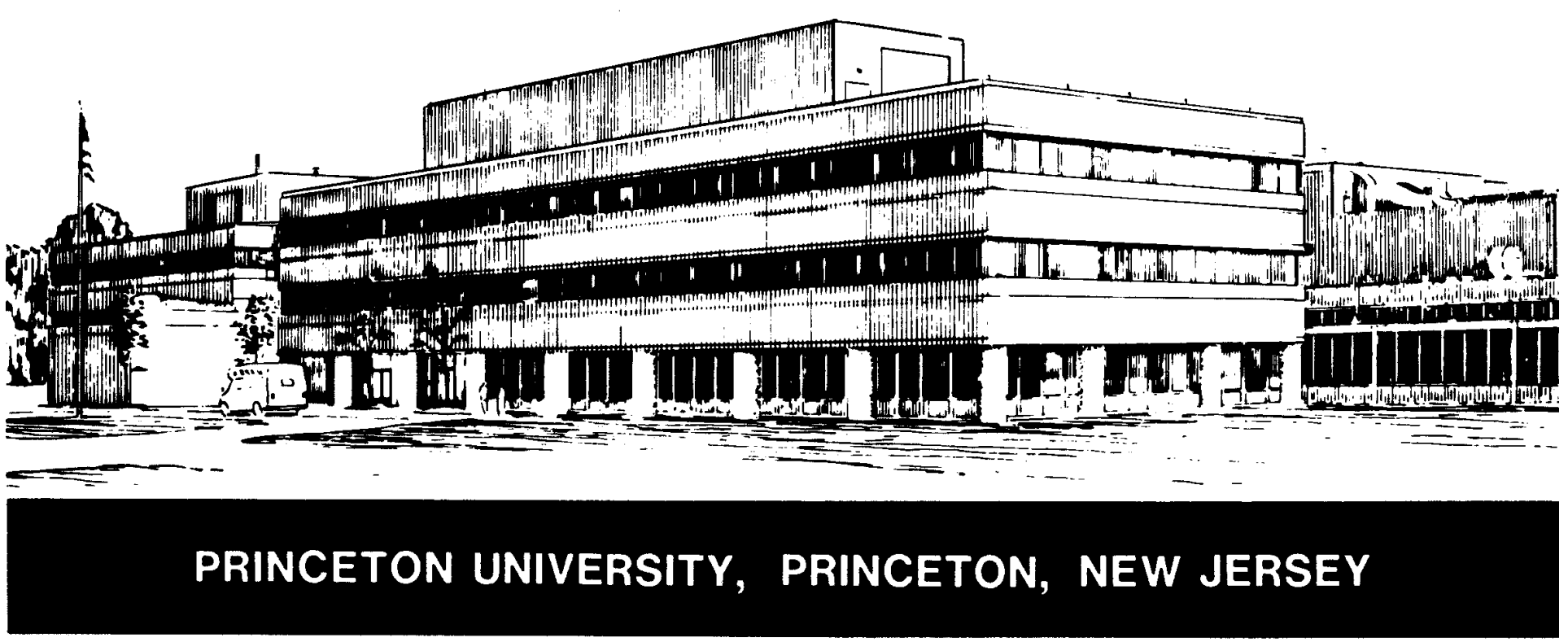




\section{PPPL Reports Disclaimer}

This report was prepared as an account of work sponsored by an agency of the United States Government. Neither the United States Government nor any agency thereof, nor any of their employees, makes any warranty, express or implied, or assumes any legal liability or responsibility for the accuracy, completeness, or usefulness of any information, apparatus, product, or process disclosed, or represents that its use would not infringe privately owned rights. Reference herein to any specific commercial product, process, or service by trade name, trademark, manufacturer, or otherwise, does not necessarily constitute or imply its endorsement, recommendation, or favoring by the United States Government or any agency thereof. The views and opinions of authors expressed herein do not necessarily state or reflect those of the United States Government or any agency thereof.

\section{Availability}

This report is posted on the U.S. Department of Energy's Princeton Plasma Physics Laboratory Publications and Reports web site in Calendar Year 2000. The home page for PPPL Reports and Publications is: http://www.pppl.gov/pub_report/

DOE and DOE Contractors can obtain copies of this report from:

U.S. Department of Energy

Office of Scientific and Technical Information

DOE Technical Information Services (DTIS)

P.O. Box 62

Oak Ridge, TN 37831

Telephone: (865) 576-8401

Fax: (865) 576-5728

Email: reports@adonis.osti.gov

This report is available to the general public from:

National Technical Information Service

U.S. Department of Commerce

5285 Port Royal Road

Springfield, VA 22161

Telephone: $1-800-553-6847$ or

(703) $605-6000$

Fax: (703) 321-8547

Internet: http://www.ntis.gov/ordering.htm 


\title{
Effect of Plasma Rotation on Sawtooth Stabilization by Beam
}

\section{Ions}

\author{
N. N. Gorelenkov ${ }^{1}$, M. F. F. Nave ${ }^{2}$, R. Budny ${ }^{1}$, C. Z. Cheng ${ }^{1}$ \\ G. Y. Fu ${ }^{1}$, J. Hastie ${ }^{3}$, J. Manickam ${ }^{1}$, W. Park ${ }^{1}$ \\ ${ }^{1}$ Princeton Plasma Physics Laboratory, Princeton, NJ, 08543, USA; ${ }^{2}$ Associação \\ EURATOM/IST, Centro de Fusão Nuclear, 1096 Lisbon, CODEX, Portugal; \\ ${ }^{3}$ EURATOM/UKAEA Fusion Assoc., Culham Science Centre, Abingdon, Oxfordshire,
} OX14 3DB, UK

The sawtooth period in JET ELM-free H-Mode plasmas is increasing with Neutral Beam Injection (NBI) power. For injected power $P_{N B I}>12 M W$ no large sawtooth crash is observed during the ELM-free period. However, as the edge stability is improved and external kink modes and ELMs are delayed, a possible sawtooth crash at a high plasma beta becomes a concern. In JET DT experiments, delaying sawteeth was found to be crucial in the quest for high fusion power [1]. Fast particles are known to provide stabilizing effect on sawteeth (see for example[2]), however, sawtooth stabilization by NBI ions is not clearly understood, since NBI ions are usually not "fast" enough to stabilize the $m / n=1 / 1$ internal kink mode which is believed to cause the crash. In order to understand the observed sawteeth stabilization in tokamak experiments with NBI heating, the internal kink $m / n=1 / 1$ mode stability of JET plasmas was modeled using the NOVA-K code[3], which is also benchmarked with the nonperturbative version of NOVA[4] and the M3D code[5]. Comparison of $m / n=1 / 1$ mode stabilization by NBI ions in JET and TFTR and application of the nonlinear $\omega_{*}$-stabilization criteria[6] is given.

\section{Code description and benchmarking}

To study the effects of the $1 / 1$ internal kink mode stabilization by NBI ions we will use the recently improved perturbative code NOVA-K, which computes quadratic form terms including hot particles

$$
\delta K=\delta W \equiv \delta W_{M H D}+\delta W_{k h}, \delta K=\omega^{2} \int \rho \xi^{2} d^{3} r .
$$

and the $1 / 1$ mode structure without fast particles. Unlike the ideal MHD case, where $\delta K \sim \omega^{2}$, we will make use of the assumption that $\delta K \sim \omega$, which produces the following dispersion relation[4] to account for the fast particle kinetic potential energy

$$
-i \omega\left(1+\frac{\omega_{T}^{2}}{\omega_{s}^{2}-\omega^{2}}\right)=\gamma_{M H D}\left(1+\frac{\gamma_{h}}{\gamma_{M H D}}\right), \frac{\gamma_{h}}{\gamma_{M H D}}=\frac{\Re \delta W_{k h}}{\delta K},
$$

where $\omega_{T}^{2}=2 \gamma_{s} P_{c} \kappa / \rho, \omega_{s}^{2}=(1 / 2) \gamma_{s} \beta_{c} \omega_{A}, \kappa$-curvature, $\gamma_{s}=5 / 3, \rho$ is plasma mass. One can see that the real part of the fast particle contribution to the potential energy $\Re \delta W_{k h}$ can give stabilization and is computed in NOVA-K according to the following formula [3], which includes particle finite orbit width (FOW) and finite Larmor radius (FLR) effects

$$
\delta W_{k h}=-(2 \pi)^{2} e_{\alpha} c \int d P_{\varphi} d \mu d \mathcal{E} \tau_{b} \sum_{m, m^{\prime}, l} \frac{X_{m, l}^{*}\left(\omega-\omega_{*}\right) X_{m^{\prime}, l}}{\omega-\overline{\omega_{d}}} \frac{\partial F_{h}}{\partial \mathcal{E}},
$$

where the integration is performed over the particle phase space $P_{\varphi}, \mu \mathcal{E}$ in general tokamak geometry, $\tau_{b}$ is the particle bounce time, $X_{m, l}$ gives wave - particle interaction power exchange, $F_{h}$ is the fast particle equilibrium distribution function, $\omega_{*}=-i \frac{\partial F / \partial P_{\varphi}}{\partial F / \partial \mathcal{E}} \frac{\partial}{\partial \varphi}$, and $\omega_{d}$ is particle toroidal drift frequency. One can see from Eq.(3) that one needs $\omega<\omega_{d}$ for the stabilization to occur. In other words, since the $1 / 1$ mode frequency is associated with plasma diamagnetic frequency $\omega_{*}$ and $\omega_{d} \sim \mathcal{E}$, particles should be energetic enough 
$\omega_{*}<\omega_{d}$ to provide the stabilization. The expression Eq.(3) can be modified to include the effect of plasma rotation on fast particle contribution by simply substituting the mode frequency $\omega \rightarrow \omega-\Omega_{E}(\psi)$, where $\Omega_{E}(\psi)$ is plasma toroidal rotation as a function of poloidal flux. This can be shown more rigorously [7]. The effect of the rotation coming from the denominator is dominant.

We have performed the benchmark of NOVA-K with its previous nonperturbative version NOVA-2 and with M3D codes for one particular case with circular plasma cross section and the following plasma parameters: major radius $R_{0}=2.62 \mathrm{~m}$, minor radius $a=0.95 \mathrm{~m}$, plasma central beta $\beta_{p l}(0)=5 \%$, fixed total beta on Fig.1 $\left(\beta_{p l}+\beta_{h}=\right.$ const $)$, and toroidal magnetic field $B=4.45 T$. Deuterium hot slowing down ions with cutoff velocity $v_{h}=10^{9} \mathrm{~cm} / \mathrm{sec}$, ratio to central Alfvén velocity $v_{h} / v_{A 0}=1$, major radius to particle Larmor radius ratio $R / \rho_{h}=55.6$. The NOVA-2 code is a non-perturbative code, which calculates the mode structure with fast particles included in the quadratic form. M3D is a non-linear code, with fast particles included. One can see that the eigenfrequency and the growth rates of fishbone branch are

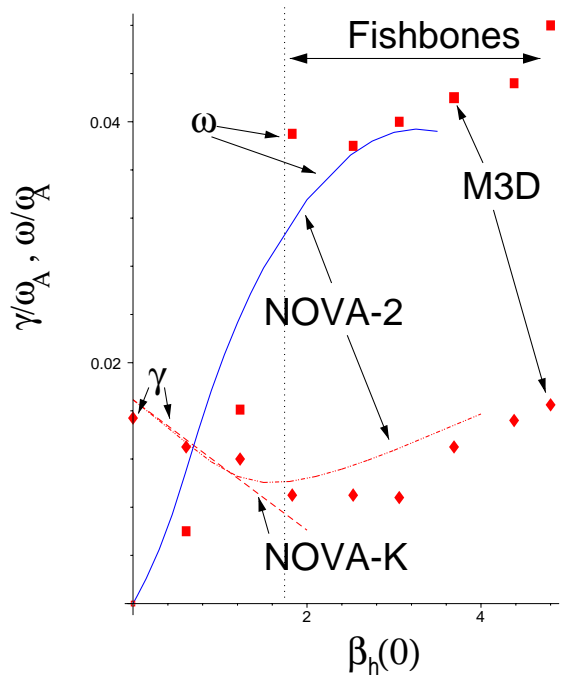

Figure 1: Codes benchmark reproduced by NOVA-2 and M3D. Linear stabilization phase of $m / n=1 / 1$ kink mode agrees for three codes. Fishbone frequency is of order of particle toroidal precession drift frequency $\omega_{d r \varphi} / \omega_{A 0}=0.49$, where $\omega_{A 0}=v_{A 0} / R$.

\section{Properties of Fast Particle Stabilization of $m / n=1 / 1$ mode in JET.}

JET high performance shot \#42976 at $t=13.3 \mathrm{sec}$ was chosen to study the properties of hot particle stabilization of $1 / 1$ mode. TRANSP was used to provide plasma parameters, which in this case were $B=3.7 T, R_{0}=2.92 \mathrm{~m}, a=0.94 \mathrm{~m}, P_{N B I d}=11.9 \mathrm{MW}, P_{N B I t}=$ $10.5 M W, P_{I C R H}=3.1 M W$. Summary of the basic calculations for stability study by NOVA-K with sheared plasma rotation velocity is given in the following table with growth rates contributing to the dispersion Eq. (2):

\begin{tabular}{|c|c|c|c|c|c|}
\hline & MHD & ICRH, $\lambda_{0} \equiv R / R_{0}$ & NBI-D & NBI-T & $\alpha$-particle \\
\hline Distribution & & $\begin{array}{c}\text { Maxw., Res. } \\
\text { layer at } \lambda_{0}=0.96\end{array}$ & Slow.Down & Slow.Down & Slow.Down. \\
\hline Energy, $k e V$ & & $\left(T_{H}=\right) 200$ & 76 & 150 & 3520 \\
\hline$\beta_{0}, \%$ & 5.81 & 0.65 & 0.25 & 0.44 & 0.6 \\
\hline$\gamma / \omega_{A}, \%$ & 1.5 & -0.9 & -0.07 & -0.17 & -0.53 \\
\hline
\end{tabular}

In the NOVA-K analysis the $1 / 1$ mode is assumed to rotate with a frequency equal to the plasma rotation frequency at the $q=1$ surface while it has a global structure and can interact with fast particles at different minor radii.

We have found that the FLR effects are negligible for fast particle stabilization, while FOW effects are small. FOW is reducing $\alpha$-particle and ICRH contributions by $\sim 20 \%$, while only slightly modifying the NBI term in Eq.(2). The distribution function of NBI ions needs special calculation and for the sake of simplicity is assumed to be slowing down in velocity and Gaussian in pitch angle $F_{b \lambda}=\exp \left(-\left(\lambda-\lambda_{0}\right)^{2} / \Delta \lambda^{2}\right)$, where $\lambda=\mu B / \mathcal{E}$. The 
geometry of the injection suggests that NBI ions are mostly passing particles. However, such effects as beam finite cross section, finite toroidicity, FOW, FLR, and Coulomb collisions can produce broad pitch angle distributions[8]. We used $\lambda_{0}=0.3$ and $\Delta \lambda=0.7$

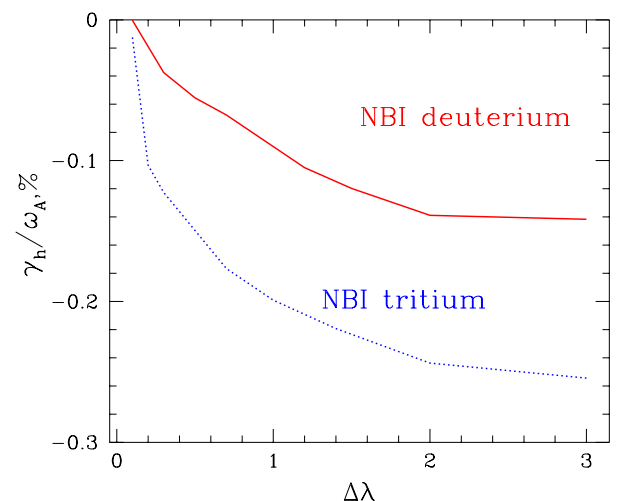

Figure 2: NBI stabilization dependence on the width of the pitch angle distribution. tion to the stabilization of $1 / 1$ mode. This is due to the fact that ICRH ions are all trapped, while their beta is comparable with $\alpha$-particle beta.

The effect of the plasma rotation is modelled by prescribing the toroidal co-current rotation velocity in the form $\Omega_{\varphi}=2.15(1-\psi)^{0.75}+0.05,10^{5} \mathrm{rad}^{-1}$, which is computed by TRANSP. We study this effect on different plasma species introducing the rotation enhancement factor as shown in Fig.4(left). The stabilization of $1 / 1$ mode is sensitive to the toroidal sheared rotation if its toroidal precession is comparable with the variation of the rotation within the $q=1$ surface $\omega_{d \varphi} \sim r \partial \Omega_{\varphi} / \partial r$. Hence, the rotation is less important for ICRH ions and $\alpha$-particles then for beam ions, which have much smaller precession frequency. The co-rotating plasma with the velocity shear supports the stabilization ef-

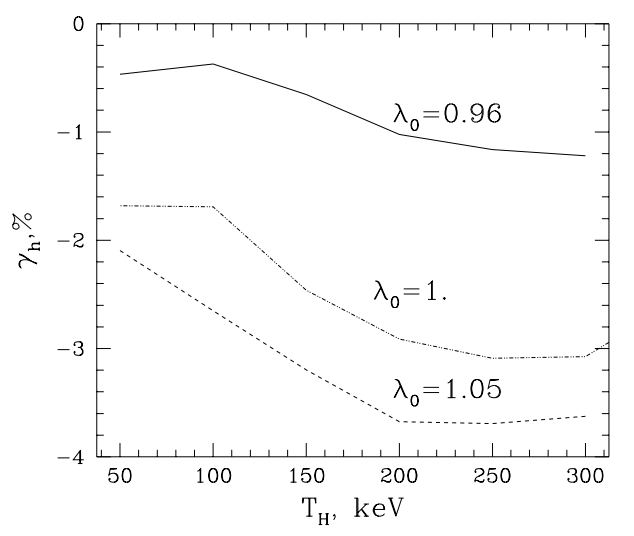

Figure 3: ICRH ion stabilization dependence on their temperature for different resonance layer positions. fectively because it increases particle energy by providing extra toroidal precession.

\section{$3 \quad$ JET NBI $\alpha$-particle experiments}

Five similar JET discharges with different tritium concentrations were analyzed using the NOVA-K code. As the tritium concentration increases, both the calculated 1/1 mode damping rate and the period between the sawteeth $\tau_{\text {saw }}$ increase. The analysis indicates that such "mass" effect come from increasing beam ion beta:

$$
\gamma_{h} \sim \beta_{h N B I} \sim S_{N B I} \tau_{s e}
$$

where the slowing down time is proportional to the beam ion mass $\tau_{s e} \sim m_{N B I}$. The results are presented on Fig. 4(right). Analysis of the co-rotating plasma shows reduction of the stabilization from fast particle for given discharges. Slightly better correlation between the measured sawtooth period and the NBI damping rate is observed in rotating plasma.

\section{Linear vs. Nonlinear $\omega_{*}$-stabilization.}



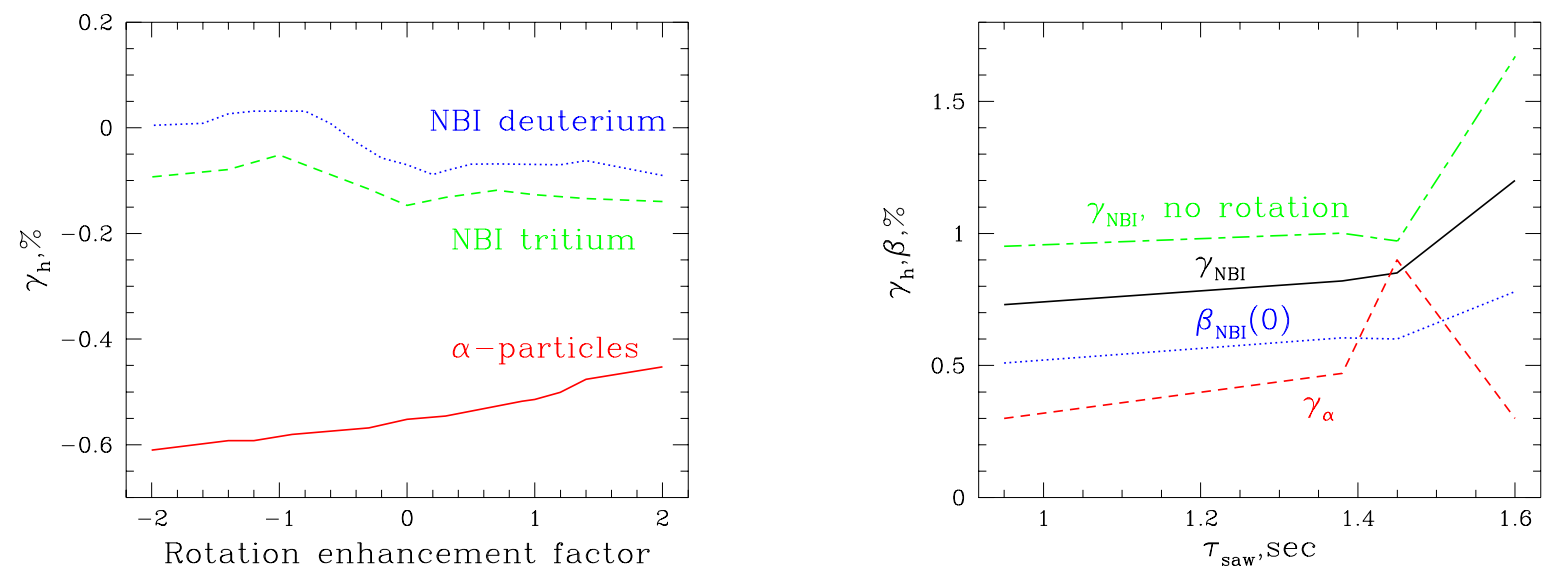

Figure 4: Effect of plasma rotation on the NBI and $\alpha$-particle stabilizing contributions (left) and analysis of the NBI heated $\alpha$-experiments on JET.

TFTR demonstrated nonlinear $\omega_{*}$-stabilization in supershots[9]. It was shown that the stability criteria works well in the conditions when $\omega_{*}$ is larger than the ideal MHD $1 / 1$ mode growth rate:

$$
r_{1} q_{1 \text { crit }}^{\prime} \equiv 1.4\left(\frac{m_{i}}{2 m_{p} Z_{e f f}}\right)^{1 / 6} \beta_{1}^{2 / 3}\left(\frac{\left|n_{e}^{\prime}\right| R}{n_{e}}\right)^{2 / 3}\left(\frac{\left|p^{\prime}\right| R}{p}\right)^{1 / 3}>r_{1} q_{1}^{\prime} \text {. }
$$

Analysis of three TFTR shots shown in Tab.1 shows smaller effect from NBI than in JET discharges: in JET plasma for the analyzed NBI only shots $r_{1} q_{1 \text { crit }}^{\prime}<r_{1} q_{1}^{\prime} \Rightarrow$ small

\begin{tabular}{|l|c|c|c|c|c|}
\hline & sawtooth & $\gamma_{b} / \omega_{A}, \%$ & $\beta_{b 0}, \%$ & $r_{1} q_{1 \text { crit }}^{\prime}$ & $r_{1} q_{1}^{\prime}$ \\
\hline$\# 65611$ & no & 0.29 & 2.2 & 0.72 & 0.29 \\
\hline$\# 65612$ & yes & 0.2 & 1.05 & 0.359 & 0.396 \\
\hline$\# 68262$ & NBI suppressed & 0.3 & 1.2 & 0.56 & 0.29 \\
\hline
\end{tabular}

Table 1: TFTR 1/1 stabilization modeling results, where $\gamma_{M H D} / \omega_{A} \simeq 3-8 \%$. $\omega_{*}$-stabilization effect. On the other hand NBI ions, supported by sheared rotation can provide stabilization of $1 / 1$ internal kink mode in JET.

\section{Summary}

Stabilizing effects on 1/1 internal kink mode by NBI hot ions is demonstrated for JET NBI alpha experiments in high performance discharges. Plasma sheared rotation, which is strong in JET, reduces the stabilizing effect of NBI ions on the growth rate of $1 / 1$ internal mode. Alpha particles offer a strong contribution to the sawtooth stabilization in high fusion plasma. Mass dependence of fast beam ion contribution to $\delta W$ is shown to be consistent with the increased sawtooth period for experiments with larger concentration of tritium NBI. While $\omega_{*}$-stabilization model by L.Zakharov appears to be consistent with TFTR sawtooth suppression in supershots, it cannot explain the increased sawtooth period with increased NBI ion beta, because of low $\omega_{*}$ in JET experiments.

\section{References}

[1] M. F. F. Nave et.al., in Controlled Fusion and Plasma Physics (Proc. 25th Eur. Conf. Praha, 1998), Vol. 22C, European Physical Society, Geneva (1998) 365.

[2] F. Porcelli et.al., Plasma Phys. Control Fusion 38, 2163 (1996).

3. N. N. Gorelenkov, et.al., Phys.Plasmas 6, 2802 (1999).

4 C. Z. Cheng, Phys. Reports, 211, 1 (1992).

5 W. Park, S. E. Parker, M. Chance et.al. Phys. Fluids B 4, 2033 (1992).

6 L. E. Zakharov, B. Rogers, and S. Migliuolo, Phys. Fluids B 5, 2498 (1992).

7 J. P. Graves, Ph.D. Thesis, University of Nottingham (1999).

8 R. Budny et.al., Nucl. Fusion, 341247 (1994).

[9] F. Levinton et.al., Phys. Rev. Lett. 
The Princeton Plasma Physics Laboratory is operated by Princeton University under contract with the U.S. Department of Energy.

\author{
Information Services \\ Princeton Plasma Physics Laboratory \\ P.O. Box 451 \\ Princeton, NJ 08543
}

Phone: 609-243-2750

Fax: 609-243-2751

e-mail: pppl_info@pppl.gov

Internet Address: http://www.pppl.gov 